\title{
Traffic Light Reporter for Genome Engineering
}

\section{Kawalpreet K Aneja*}

Biology Teacher for School District of Philadelphia and Postdoctoral Fellow at University of Pennsylvania and Independent Scholar, Philadelphia, PA, USA

*Corresponding Author: Kawalpreet K Aneja, Biology Teacher for School District of Philadelphia and Postdoctoral Fellow at University of Pennsylvania and Independent Scholar, Philadelphia, PA, USA.
Received: May 03, 2020

Published: August 18, 2020

(C) All rights are reserved by Kawalpreet $\mathbf{K}$ Aneja.
Gene editing depends upon the cell's endogenous DNA repair pathways. Upon DNA double-strand break, two known repair pathways are enacted: nonhomologous end-joining (NHEJ) and homologous recombination (HDR), among others. The HDR pathway works only in the presence of an exogenous homologous template. Is it possible to determine which pathway is activated in a cell after gene disruption (GD)? Is it NHEJ or HDR? Certo., et al. 2011 [1] published an article on the Traffic Light Reporter (TLR) that can distinguish between NHEJ and HR events. Traffic light reporter system is designed such that cells generate flow-cytometric readout of HDR-mediated gene targeting or mutagenic NHEJ-mediated gene disruption occurring at an individual DNA breakpoint, which is extremely exciting!

A TLR is made up of two fluorescence reporters, enhanced GFP and monomeric Cherry separated by self-cleaving T2A peptide. By design, the $e G F P$ coding sequence contains an alternative +3 reading frame and the T2A linker that enables the downstreamencoded mCherry to escape the degradation of a faulty protein GFP encoded in +3 reading frame of $e G F P$. So, this way if functional $e G F P$ is restored by HDR, GFP can be detected by a flow cytometer. However, in case of NHEJ, a gibberish GFP protein from the +3 frame would be created, leading to Cherry expression.

A single copy of the TLR containing an I-SceI target site in eGFP sequence was integrated into the genome of HEK293T cells, named HEK293T TLR-Sce. I-SceI is a mitochondrial intron encoded endonuclease from Saccharomyces cerevisiae that targets an 18-bp sequence TAGGGATAACAGGGTAAT. I-SceI was expressed with or without a donor template in HEK293T TLR-Sce cells. Cells transduced with both I-SceI and donor template lentivirus yielded Cherry+ or GFP+ cells. Cells transduced with I-SceI lentivirus alone resulted in cells expressing Cherry+, indicative of NHEJ at the reporter locus. Sequence analysis confirmed that Cherry+ cells were generated by a variety of mutagenic events at the I-SceI target site that resulted in frameshifts to the +3 reading frame. These frameshifts represented about one-third of all the mutagenic events. Certo., et al. [1] figure 1 confirms that sufficient cells can be sorted by flow cytometry to derive single-cell clones in GFP or Cherry gates.

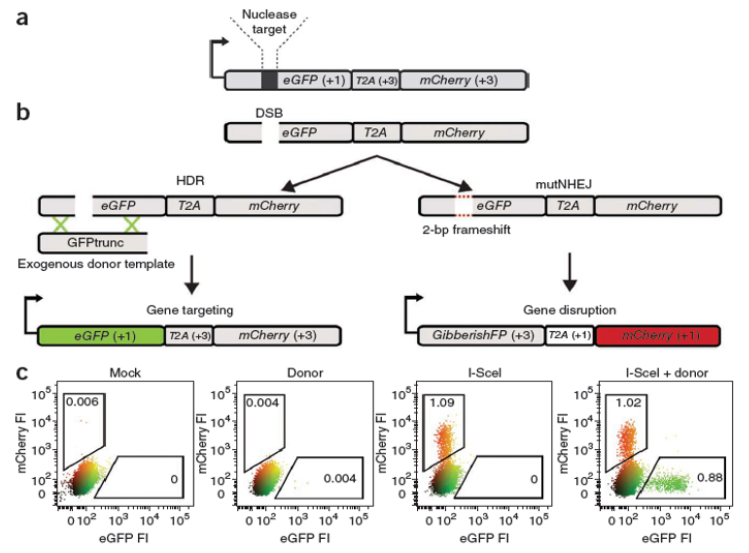

Figure 1: Genome design or engineering by traffic light reporter system [1].

a. TLR made up of two fluorescence reporters, GFP and Cherry separated by self-cleaving T2A peptide.

b. Stably transfected HEK293T TLR-Sce cell line with integrated TLR containing I-SceI targeting site was made. Cells expressing GFP or CHERRY can be sorted by flow cytometry to derive single-cell clones. In HR case, a functional enhanced GFP ( $e G F P$ ) open reading frame is restored by the exogenously provided donor template to signal gene targeting. And in NHEJ case a frameshift places a monomeric m(Cherry) coding sequence in-frame to signal gene disruption. 
Certo., et al. [1] also tested the effect of an increasing dose of I-SceI or donor template, both delivered with a single lentivirus. They found a dose-dependent increase in the total number of repair events, with an increasing fraction of events derived from the HDR pathway. The authors then went to compare the TLR readout between double-strand and single-strand breaks. They created another TLR cell line containing an I-AniI nuclease target site (HEK293T TLR-Ani) and compared between nuclease and nickase activity of AniI variants. Anil nuclease makes double-strand while nickase makes single-strand breaks. I-Anil nickase showed 100fold fewer mutations than the I-Anil nuclease but nickase showed a preference for HDR over NHEJ.

The DNA damage response is highly regulated primarily by phosphorylation. PRKDC encodes the catalytic subunit of the DNAdependent protein kinase (DNA-PK) and helps in DNA repair and recombination. HEK293T TLR-Sce cells pretreated with siRNA to knockdown PRKDC were transduced with both I-SceI-T2A-IFP lentivirus and donor integration-deficient lentivirus showed a tenfold increase in gene targeting or HDR events with only a modest associated increase in NHEJ events. A consistent increase in gene targeting events was seen after PRKDC knockdown in multiple TLR clones.

This paper shows a promising system for fluorescent readout of the repair pathway. We can use this system by cloning Cas 9 target guide RNA sequence in $e G F P$ in place of I-SceI target. This system can also be used as a control by cloning a gene of choice in place of $e G F P$ with mCherry expression. In conclusion, TLR can be used more than just a reporter. TLR is an excellent reporter for genome designing and engineering.

Based upon TLR there is a new reporter system, which can separate actively transcribing cells from repressed cells [2]? And it is named as the "Active/Repressed TLR (AR-TLR)". In AR-TLR, near infra-red fluorescent (iRFP) protein and T2A were attached upstream of $e G F P$ and under the control of the spleen focus forming virus promoter (SFFV). So, iRFP+ and iRFP- cells representing chromatin active or repressed states can be separated via flow cytometer. Furthermore, another reporter was made for the detection of single-stranded annealing assay-SSA-TLR, single-stranded annealing is common in genomic repetitive regions. It was done by cloning 5' - iRFP arm lacking C-terminal 38 amino acids and 3'-iRFP arm missing N-terminal 25 amino acids [2]. Upon double-stranded DNA break, if resection reveals 762 base pairs homology between two arms, it makes them anneal. This annealing of two arms allows the expression of iRFP and confirms that SSA just happened in cells. These reporters are extremely valuable for detecting cell cycle, cell division and cell signaling pathways and would be essential tools for genome editing or genome surgery in the future.

\section{Bibliography}

1. Michael T Certo., et al. "Tracking genome engineering outcome at individual DNA breakpoints". Nature Methods 8 (2011): 671-676.

2. Ryan Kuhar., et al. "Novel fluorescent genome editing reporters for monitoring DNA repair pathway utilization at endonuclease-induced breaks". Nucleic Acids Research 42.1 (2014): e4.

\section{Assets from publication with us}

- Prompt Acknowledgement after receiving the article

- Thorough Double blinded peer review

- Rapid Publication

- Issue of Publication Certificate

- High visibility of your Published work

Website: www.actascientific.com/

Submit Article: www.actascientific.com/submission.php

Emaill us: editor@actascientific.com

Contact us: +919182824667 